\title{
The Assessment of the Suitability of Mariveles Public Beachfront for Public Bathing: A Revisit Study
}

\author{
Erdy S. Aganus, Lawrence Charlemagne G. David \\ Maritime Academy of Asia and the Pacific \\ Mariveles, Bataan, Philippines \\ aganuserdy@yahoo.com; lawrencecharlemagnedavid@ieee.org
}

\section{Extended Abstract}

Aim: To conduct water quality assessment in the public beachfront in the urban area of Mariveles town in Bataan, Philippines and compare the results with the previous evaluation.

Methods: Four public bathing sites were revisited. Three water samples in each site were collected during the premonsoon season (March 2019) using sterilized bottles for three consecutive weeks. The samples were tested for three bacteriological parameters, namely, faecal coliform, total coliform and heterotrophic plate count. Total coliforms and faecal coliforms have been historically used to establish bathing water quality.

Results: As basis, the researchers used the latest version of the Philippine National Standards for Drinking-Water, which also complies with the standards set by the World Health Organization [1]. Both faecal coliform and total coliform were greater than 8.0 MPN/100 mL in most of the samples. Heterotrophic plate count (in CFU/100 mL) was highest in the location near the outlet of Santol river $(M=2138.9, S D=1664.7)$ and lowest for Matel beach $(M=281.11, S D=120.25)$. Using a one-way analysis of variance, the study showed that there was no significant difference in the HPC of the four sampling locations in the present study. With a one-sample T-test, the researchers found that the water samples did not meet the standards set by the DOH and WHO. Compared with the previous study, a significant decrease in average HPC was observed in the location near the breakwater. Although there was also a decrease in the mean HPC of the location near Santol river and London Drug, the reduction was insignificant. On the contrary, an increase in average HPC was seen on Matel beach.

Conclusions: The locations are still classified as high risk mainly due to faecal coliform concentrations, as well as the other two parameters. Public bathers can face gastrointestinal risks when they (accidentally) drink water from these locations. Thus, the water from the studied locations is not recommended for public bathing.

Significance of the Study: The results presented in this research can be used as data in tracking the progress of the recent implementation of policies made by the local government of Mariveles geared towards improving the water quality in its town.

\section{References}

[1] M. Lomboy, J. R. d. Dios, B. Magtibay, R. Quizon, V. Molina, V. Fadrilan-Camacho, J. See, A. Enoveso, L. Barbosa and A. Agravante, "Updating national standards for drinking-water: a Philippine experience," Journal of Water and Health, pp. 288-295, 2017. 